\title{
LA HISTORIA DE LAS MATEMÁTICAS EN LOS CURSOS DE EDUCACIÓN BÁSICA EN PORTUGAL: UNA REFLEXIÓN PARA LA FORMACIÓN DEL PROFESORADO
}

\author{
Hélder Pinto ${ }^{1}$ \\ helder.pinto@gaia.ipiaget.pt \\ Cecília Costa ${ }^{2}$ \\ mcosta@utad.pt \\ ${ }^{1}$ Instituto Piaget, RECI - Research in Education and Community Intervention e CIDMA \\ ${ }^{2}$ Universidade de Trás-os-Montes e Alto Douro e CIDTFF \\ Há tantos pensadores que nunca aprendem \\ E há quem insista sempre em aprender \\ Mas não quer pensar \\ Optimista Céptico (Jorge Palma)
}

Recibido: 07/10/2019 Aceptado: 06/01/2020

\begin{abstract}
Resumen
La Historia de las Matemáticas (HM) es una herramienta que puede ser muy útil en un contexto educativo. Sin embargo, la HM es un cuerpo de saber muy extenso y es necesario reflexionar cual HM debe ser enseñada en la formación inicial de los profesores en los primeros años de escolaridad. En este artículo hacemos esta reflexión tratando de justificar qué contenidos HM son realmente esenciales para los profesores y para sus futuras prácticas profesionales. En nuestra opinión, los contenidos de HM deben centrarse en los temas: sistemas de numeración de diversos pueblos, diferentes algoritmos para realizar operaciones aritméticas y resolver ecuaciones, tópicos de la geometría, así como el conocimiento de varios puntos de HM local/nacional. También damos una breve descripción de lo que son los cursos de Educación Básica en Portugal, prestando especial atención a las matemáticas que se enseñaban en estos cursos. Por otro lado, también presentamos varias referencias a estudios internacionales donde se atestigua la importancia de usar HM en el aula.
\end{abstract}

Palabras clave: Educación básica Formación del profesorado, historia de las matemáticas, historia de las matemáticas como herramienta en un contexto educativo.

\section{HISTORY OF MATHEMATICS IN PORTUGUESE INITIAL BASIC TEACHER EDUCATION PROGRAMS: A REFLECTION FOR TEACHER TRAINING}

\begin{abstract}
History of Mathematics (HM) is a tool that can be very useful in an educational context. However, HM is a very broad corpus of knowledge and it is necessary to reflect on what HM should be taught in the initial basic teacher education programs. In this paper, we make this reflection by trying to justify which HM contents are truly essential for teachers and their future professional practices. In our opinion, HM contents should focus on the following topics: diverse ancient peoples numbering systems, different algorithms for performing arithmetic operations and solving equations, geometry topics, as well as knowledge of various local/national HM aspects. We also give a brief description of Portuguese initial basic teacher education programs, giving special focus
\end{abstract}


on the mathematics syllabus in these programs. And we present several references to international studies that show the importance of using HM in the classroom context.

Keywords: Training of elementary school teachers, history of mathematics, history of mathematics as a tool in educational context.

\title{
A HISTÓRIA DA MATEMÁTICA NOS CURSOS DE EDUCAÇÃO BÁSICA EM PORTUGAL: UMA REFLEXÃO PARA A FORMAÇÃO DE PROFESSORES
}

\begin{abstract}
Resumo
A História da Matemática (HM) é uma ferramenta que poderá ser muito útil em contexto educativo. Contudo, a HM é um corpo de saber muito extenso e é necessário refletir qual a HM que deverá ser ensinada na formação inicial de professores dos primeiros anos de escolaridade. Neste artigo fazemos essa reflexão tentando justificar quais os conteúdos de HM verdadeiramente essenciais para os professores e para as suas práticas profissionais futuras. Na nossa opinião, os conteúdos de HM deverão centrar-se nos temas: sistemas de numeração de diversos povos, diferentes algoritmos para realizar operações aritméticas e resolver equações, tópicos de geometria, bem como o conhecimento de vários pontos de HM local/nacional. Fazemos ainda uma breve descrição do que são os cursos de Educação Básica em Portugal, dando especial enfoque nas matemáticas lecionadas nesses cursos. Por outro lado, apresentamos também várias referências a estudos internacionais onde se atesta a importância da utilização da HM em contexto de sala de aula.
\end{abstract}

Palavras- chave: Formação de professores do ensino básico, história da matemática, história da matemática como ferramenta em contexto educativo.

\section{Introdução}

A ideia de que a História da Matemática (HM) pode contribuir para a formação de professores não é nova e continua a ser defendida atualmente (Arcavi, Bruckheimer, \& Ben-Zvi, 1982; Isaacs, Ram, \& Richards, 2000; Mosvold, Jakobsen, \& Jankvist, 2014; Mendes, 2015; Mendes \& Chaquiam, 2016).

Neste artigo de reflexão pretendemos ilustrar e discutir propostas de tópicos de HM que podem contribuir para uma melhor compreensão da matemática que os futuros professores (portugueses) de $1 .^{\circ}$ e $2 .^{\circ}$ ciclos do ensino básico terão de ensinar.

Subjacente à reflexão aqui apresentada estão quatro objetivos gerais relativos ao domínio da HM por um professor do ensino básico que consideramos essenciais, a saber:

1. Situar a evolução dos conceitos matemáticos ao longo da História com enfoque nos sistemas de numeração, nas operações aritméticas, na resolução de equações e na geometria.

2. Associar a HM com a História da Humanidade, evidenciando os maiores avanços matemáticos com a história das grandes civilizações da 
antiguidade como os babilónios, os antigos egípcios, os gregos, os árabes, etc..

3. Conhecer a importância da matemática ao longo da História em diversas áreas como a astronomia, a navegação, a engenharia, a cobrança de impostos, etc..

4. Perspetivar a HM como uma ferramenta educativa motivacional para a sala de aula, permitindo humanizar a disciplina.

Neste artigo, descrevemos, sinteticamente, como é realizada a formação de professores do ensino básico em Portugal (cap. 1), apresentamos argumentos a favor da utilização da HM nessa formação (cap. 2) e, do extenso corpo de saber que é a HM, selecionamos tópicos e sugerimos algumas propostas dessa utilização, tendo em conta os conteúdos matemáticos que os futuros professores terão de ensinar (cap. 3).

\section{Cap 1. Os cursos de Educação Básica em Portugal}

Em Portugal, os $1 .^{\circ}$ e $2 .^{\circ}$ ciclos do ensino básico abrangem os seis primeiros anos de escolaridade (respetivamente, quatro anos mais dois) e destinam-se a alunos dos 6 aos 12 anos de idade. Aqueles que pretendem, profissionalmente, ser professores do $1 .^{\circ}$ ou do 2. ${ }^{\circ}$ ciclo do ensino básico (em Português, História e Geografia de Portugal ou em Matemática e Ciências da Natureza) têm de ter uma formação inicial de três anos numa licenciatura em Educação Básica, numa universidade ou instituto politécnico e, posteriormente, ter formação especializada através de cursos de mestrado específicos para formação de professores. Dependendo da formação especializada escolhida, poderão ou não lecionar matemática no $2 .{ }^{\circ}$ ciclo do ensino básico $\left(2 .{ }^{\circ} \mathrm{CEB}\right)$, mas podem sempre fazê-lo no $1 .^{\circ}$ ciclo do ensino básico $\left(1 .^{\circ} \mathrm{CEB}\right)$.

As licenciaturas em Educação Básica têm um cariz generalista, muito abrangente e pouco aprofundado, oferecendo formação na área da docência (português, matemática, ciências, história e geografia de Portugal e expressões), formação educacional geral, didáticas específicas e iniciação à prática profissional. Em (Galvão, Ponte, \& Jonis, 2018) encontram-se mais detalhes sobre a formação inicial de professores em Portugal. A formação matemática exigida para a candidatura a estes cursos não é a mais completa e alargada que é oferecida no ensino não superior. Sendo cursos superiores que, habitualmente, têm classificação média de entrada na universidade baixa, atraem muitos alunos que não têm boa formação matemática, nem motivação para serem professores. 
Os cursos de mestrado específicos para a formação de professores, dependendo da instituição, podem dar habilitação para a docência na Educação Pré-escolar e/ou no $1^{\circ} \mathrm{CEB}$, ou para o $1 .^{\circ} \mathrm{CEB}$ e para o $2 .^{\circ} \mathrm{CEB}$, variante de Português, História e Geografia de Portugal ou variante Matemática e Ciências de Natureza. Nestes cursos há um reforço da formação na área da docência, nas didáticas específicas e surge a prática de ensino supervisionada que incide sobre os níveis de ensino para que forma o respetivo curso. Em (Galvão \& Ponte, 2018) encontra-se uma resenha histórica e reflexão sobre os Mestrados em Ensino no contexto atual da formação de professores em Portugal. Ainda que o foco seja o panorama na Universidade de Lisboa, os contornos legais são os mesmos para as restantes instituições de formação de professores para o ensino básico.

Focando-nos na formação que importa para a reflexão aqui apresentada, no final da formação inicial e especializada, os alunos tiveram contacto com conteúdos de matemática (tradicionalmente de lógica, números e operações, geometria e medida, organização e tratamento de dados), conteúdos de didática da matemática e conteúdos de história da ciência. A abordagem explícita a aspetos de HM, existirá ou não dependendo da vontade do professor e, portanto, a frase de Mosvold, Jakobsen e Jankvist (2013):

"Despite the evidence provided for this claim by the authors [o facto de os futuros professores poderem beneficiar com a aprendizagem da HM], many mathematics teachers today still go through their training programs without ever becoming acquainted with the history of mathematics"

é aplicável no contexto português que estamos a tratar.

No próximo capítulo apresentamos argumentos a favor da importância de incluir, explicitamente, a HM na formação de professores do ensino básico.

\section{Cap 2. O papel da História da Matemática nos cursos de Educação Básica}

Nos primeiros anos do século XXI, o National Council of Teachers of Mathematics (NCTM, 2003) insistia na ideia de que os programas de formação de professores de matemática deviam incluir HM e as contribuições de diversas culturas, no sentido de preparar os futuros professores para promoverem a proficiência do aluno na HM em sete áreas de conteúdo: (1) números e operações, (2) álgebra, (3) geometria (4) estatística e probabilidade, (5) cálculo, (6) matemática discreta e (7) medida (Galante, 2014). No caso da formação de professores do ensino básico em Portugal, 
dados os conteúdos que, previsivelmente, irão lecionar, entendemos que as áreas de conteúdo (1), (2) (3) e (7) e, eventualmente a (4), seriam as essenciais.

Porquê esta insistência?

Arcavi e colegas, em 1982, sugeriram que "maybe a mathematics teacher can profit from the study of the history of mathematics" (Mosvold, Jakobsen, \& Jankvist, 2014). Desde então vários estudos têm vindo a ser feitos procurando mostrar que existem vantagens para os professores em estudar (alguns tópicos relevantes de) HM.

No seguimento das razões apresentadas por Fauvel, em 1991, para se usar a HM no ensino da matemática e, consequentemente, na formação de professores, Lui, em 2003, apresentou cinco razões que passamos a enunciar:

“A história pode ajudar a aumentar a motivação e desenvolver uma atitude positiva em relação à aprendizagem. Os obstáculos do passado no desenvolvimento da matemática podem ajudar a explicar o que os alunos de hoje acham difícil. Problemas históricos podem ajudar a desenvolver o pensamento matemático dos alunos. A história revela as facetas humanísticas do conhecimento de matemática. A história fornece aos professores um guia para o ensino" (Liu, 2003, p. 416 em Galante, 2014).

Nestas razões, fica claro que não se está a entender a "HM como objetivo" (isto é, conhecer a história real da matemática, incluindo pessoas, lugares e eventos envolvidos), mas sim, a "HM como ferramenta" (para proporcionar interesse, motivação e compreensão dos esforços envolvidos na descoberta matemática). Esta distinção é devida a U. T. Jankvist em estudos do início do séc. XXI (Mosvold, Jakobsen, \& Jankvist, 2014).

Que evidências existem da utilidade da HM como ferramenta?

Tendo em conta o modelo de Shulman (1986) para o conhecimento profissional do professor, para além das dimensões base do Conhecimento do Conteúdo e o Conhecimento Pedagógico, este autor estabeleceu uma nova dimensão do conhecimento que resulta da interseção desses dois conhecimentos: Conhecimento Pedagógico do Conteúdo. Em síntese, segundo Shulman (1986) o professor para além dos conhecimentos científicos relativos a um determinado conteúdo, deve também conhecer estratégias pedagógicas e saber adequá-las a esse mesmo conteúdo.

Como afirma Galante (2014), ensinar futuros professores sobre HM é adicionar conhecimento ao conhecimento de conteúdo de matemática e aumentar o conhecimento 
de conteúdo pedagógico e pode ser uma tarefa que valha a pena. Os resultados deste estudo indicaram que, ao permitir que os futuros professores de matemática investigassem e apresentassem tópicos de HM, estes acreditavam ter fortalecido o seu conhecimento sobre o conteúdo de matemática e consideraram ter encontrado uma nova forma para ensinar matemática aos seus futuros alunos.

Outros estudos (Jankvist, 2019; Mosvold, Jakobsen, \& Jankvist, 2014) confirmam estes resultados e referem ainda que os professores podem adquirir conhecimento sobre o conceito matemático a um nível meta e ainda que, em relação ao conhecimento pedagógico do conteúdo, a HM fornece um repertório de ideias para o professor de matemática, quanto mais não seja porque fornece exemplos autênticos de problemas matemáticos (que envolvem ideias, conceitos e métodos do passado).

Defendemos que a HM, por um lado, ajuda o (futuro) professor, enquanto aluno, a compreender melhor as ideias, conceitos e métodos matemáticos e, por outro, fornece estratégias para o (futuro) professor, quando professor, os ensinar. No entanto, a HM é um corpo de saber demasiado amplo e extenso, pelo que se torna pertinente refletirmos sobre quais os conteúdos de HM verdadeiramente essenciais para os professores e para as suas práticas profissionais futuras, é o que fazemos no capítulo seguinte.

\section{Cap 3. A História da Matemática para os cursos de Educação Básica - propostas}

A maioria dos autores de referência nesta área (por exemplo, Boyer, 1996; Katz, 2004; Eves, 1997; Struik, 1992; Estrada, Sá, Queiró, Silva \& Costa, 2000) começam as suas obras com a matemática do antigo Egipto (existem registos desde 3100 a.C.) e da Mesopotâmia (civilização iniciada por volta do quinto milénio a.C.) - note-se que existem registos escritos substanciais destas duas civilizações, em particular, na área da matemática. Embora haja registos de algumas formas rudimentares de matemática anteriores como, por exemplo, contagens de objetos em ossos, o ponto de partida centra-se nestas duas civilizações que construíram um corpo amplo de saber na área da matemática.

Os egípcios, por exemplo, tinham conhecimentos em frações e em geometria e a civilização babilónica (povo que vivia na mesopotâmia) já tinha conhecimentos tão avançados como, por exemplo, equações quadráticas e cúbicas. Depois destas duas civilizações, é possível ainda apontar vários outros povos importantes na HM como, por exemplo, os hindus, os chineses, os árabes e os gregos que também construíram um vasto conjunto de conhecimentos matemáticos e que hoje são centrais na matemática 
escolar e não só. Neste capítulo vamos focar-nos, de entre toda a extensão da HM, nos conteúdos que entendemos serão verdadeiramente úteis aos futuros professores do ensino básico. No que se segue apresentamos alguns tópicos que, em nossa opinião, nos parecem essenciais, bem como a justificação para serem pertinentes na formação de professores.

\subsection{Sistemas de Numeração}

O sistema de numeração indo-arábico atual foi criado pelos Hindus e difundido pela civilização árabe. Este sistema é utilizado em quase todo o mundo e a sua «vitória» sobre os antigos sistemas deve-se às suas «boas» propriedades matemáticas. Um processo convincente para se perceber essas «boas» propriedades do nosso sistema atual é compará-lo com os sistemas antigos e perceber as vantagens e as desvantagens de cada um deles.

Só se entende as vantagens do sistema decimal quando se tenta trabalhar com outros sistemas como, por exemplo, o sistema sexagesimal dos babilónios ou o sistema vigesimal dos maias (em rigor, o sistema de numeração maia não é totalmente vigesimal pois pretendia-se que o número 360 - o número de dias de um ano para os maias tivesse um papel fulcral no seu sistema de numeração).

Só se consegue perceber a «economia» do nosso sistema (conseguir escrever todos os números com apenas dez símbolos) quando se tenta escrever, por exemplo, o número 999 em sistemas repetitivos como era o sistema do antigo Egipto (Fig. 1).

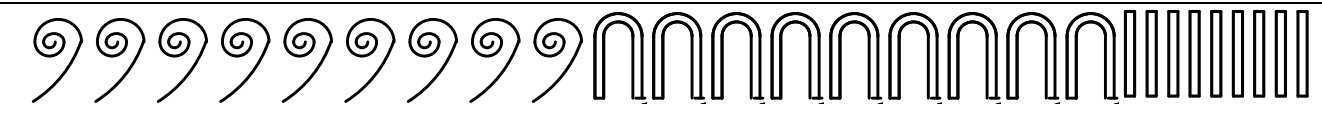

Fig. 1. O número 999 escrito no sistema de numeração do antigo Egipto

Por outro lado, ao longo da HM encontram-se vários sistemas posicionais como o nosso atual e que permitiam escrever todos os números naturais com um número finito de algarismos. Esta característica compreende-se bem, por oposição, no sistema de numeração do antigo Egipto, que era limitado superiormente: o maior número que podia ser escrito nesta numeração era o número 99.999.999 (o símbolo que representava um Sol, era usado para representar o maior número e tinha o valor de 10.000.000). Nesta questão é ainda de salientar a numeração romana pois era, em parte, posicional (IX era diferente de XI), mas também era do tipo repetitiva e limitada superiormente. Note-se ainda que muitos sistemas antigos ainda não possuíam o zero, o 
que levava a grandes dificuldades e equívocos nos sistemas posicionais - fazendo analogia com o nosso sistema atual, como distinguir os números 34 e 304 se não tivéssemos o símbolo 0 para colocar no meio das duas posições?

Assim, no nosso entender, é essencial os futuros professores do ensino básico estudarem o sistema de numeração dos antigos egípcios (muito simples e que os alunos, mesmo os mais novos, facilmente compreendem), da civilização babilónica (até porque ainda hoje se utiliza o sistema sexagesimal, por exemplo, nos relógios, temática que faz parte do ensino do primeiro ciclo em Portugal), dos romanos (numeração ainda utilizada em vários contextos), da civilização maia (ligada à astronomia e de base (quase) 20) e da antiga civilização chinesa (por exemplo, o sistema dos Tsungs e Hengs da dinastia Shang e que pode ser consultado em http://www-history.mcs.stand.ac.uk/HistTopics/Chinese_numerals.html). Na Fig. 2 apresentamos um exemplo de utilização deste assunto num manual escolar do $3^{\circ}$ ano do $1 .^{\circ} \mathrm{CEB}$.

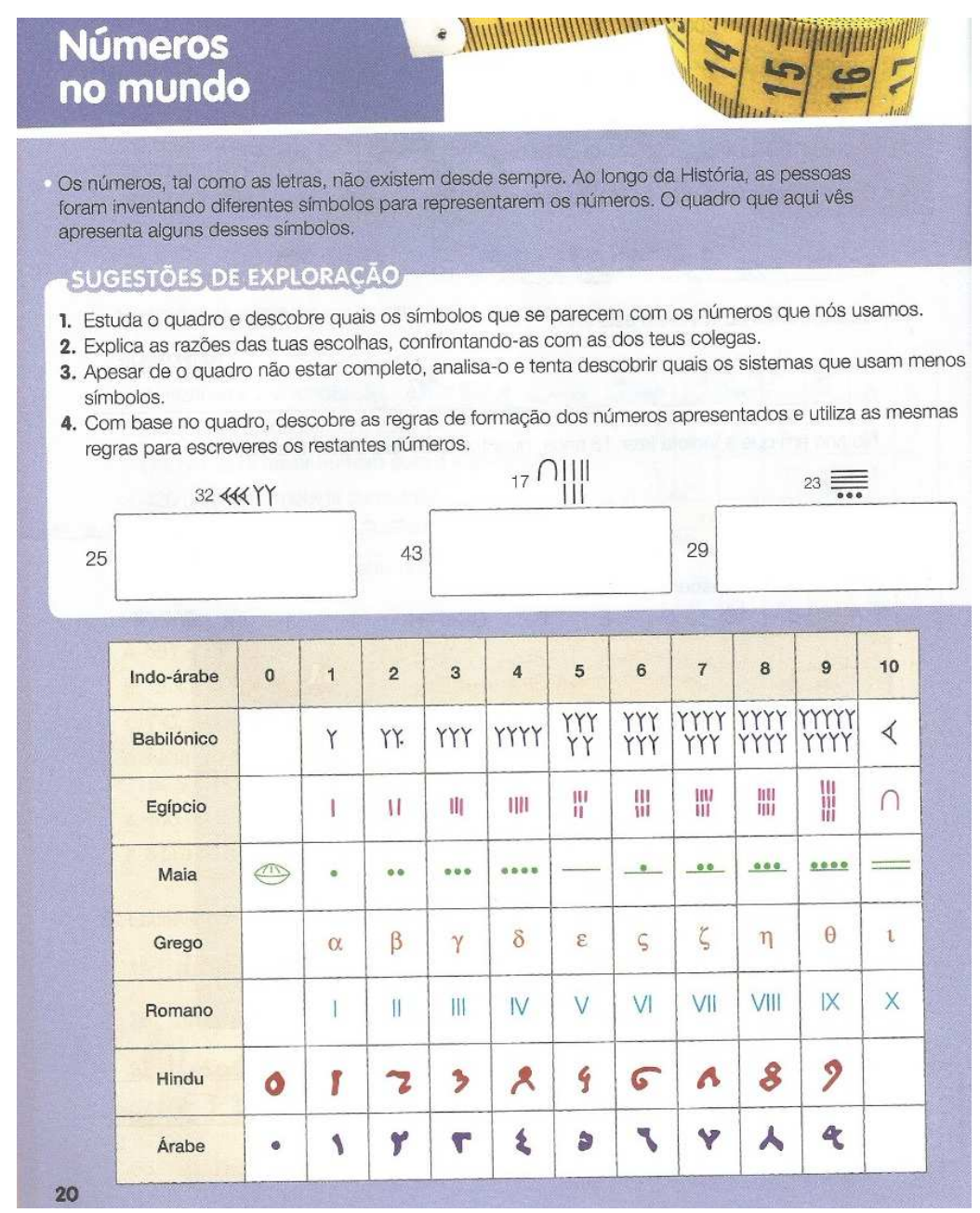

Fig. 2. Exemplo da utilização de HM num manual do $1 .{ }^{\circ} \mathrm{CEB}\left(3 .^{\circ}\right.$ ano) em Portugal (Lima, Pedroso, Barrigão, \& Santos, 2019, p. 20) 
Para além da questão matemática, estes conhecimentos permitem humanizar a matemática, mostrando-a como uma ciência em constante evolução e como o resultado do trabalho de muitas gerações e diferentes povos. Saber que os "nossos" números foram criados na Índia e que chegaram à Europa através da atuação dos árabes permite uma nova visão da matemática, percebendo que a globalização também existiu noutras eras da História e mostrando que os outros povos também deram e continuam a dar contributos científicos válidos.

\subsection{Operações aritméticas}

O ensino das quatro operações aritméticas é uma das primeiras tarefas de um professor do $1 .^{\circ} \mathrm{CEB}$. Infelizmente, muitas vezes, o ensino centra-se nos algoritmos não deixando margem para que se perceba, de facto, porque é que os algoritmos funcionam e permitem chegar ao resultado pretendido (este problema é particularmente evidente na multiplicação e na divisão).

Uma forma de retomar as operações aritméticas e a sua compreensão é ensinar aos alunos outros métodos de efetuar as operações. Ao longo da HM encontramos vários métodos que podem ser utilizados em sala de aula com facilidade e que podem ser comparados/analisados com os métodos atuais. Um dos mais conhecidos é o método de gelosia; para uma explicação de como funciona este método, consultar, por exemplo, Pinto, 2011, pp. 107-109. O método de gelosia é muito atrativo visualmente e pode ser introduzido/explicado rapidamente em sala de aula (apenas é necessário saber a tabuada para preencher a tabela e, de seguida, somar aos valores de cada diagonal, como se ilustra na Fig. 3).

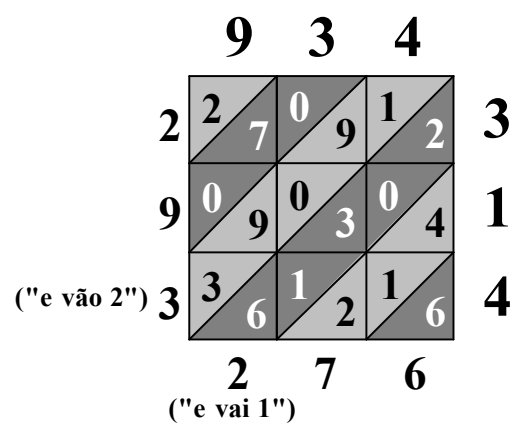

Fig. 3. Exemplo de multiplicação em gelosia: $934 \times 314=293276$.

O importante é mostrar aos alunos que o método é perfeitamente análogo ao algoritmo atual embora a apresentação seja substancialmente diferente (o que se soma nas «diagonais» em gelosia, soma-se nas «colunas» do método atual). Observe-se a 
seguir o algoritmo atual (Fig. 4) onde deixamos a indicação do que «veio de trás» em cada momento.

\begin{tabular}{llllll} 
& & & 9 & 3 & 4 \\
& & $\times$ & 3 & 1 & 4 \\
\hline & & 3 & $6+1$ & $2+1$ & 6 \\
& 0 & $9+0$ & $3+0$ & 4 & \\
2 & $7+0$ & $9+1$ & 2 & & \\
\hline 2 & 7 & $\mathbf{2 2}$ & $\mathbf{1 2}$ & $\mathbf{7}$ & $\mathbf{6}$
\end{tabular}

Fig. 4. Exemplo de multiplicação pelo algoritmo usual: $934 \times 314=293276$.

Deste modo, volta-se a rever o método atual, possivelmente sem que os alunos se apercebam que estão novamente a trabalhar as operações aritméticas essenciais.

Outro método que pode ser interessante em sala de aula é o método de multiplicação e divisão do antigo Egipto. Este método é substancialmente diferente do nosso e usa apenas duplicações sucessivas, ou seja, não exige o conhecimento das tabuadas (superiores a 2) que o nosso método exige. De facto, este método utiliza, sem o enunciar, a decomposição do multiplicador em parcelas de potências de 2 , o que é sempre possível para qualquer número natural.

De facto, para os antigos egípcios, multiplicar 19 por 11 era o mesmo que multiplicar 19 por $(1+2+8)$ e, portanto, bastava fazer as seguintes multiplicações: $19 \times 1$, $19 \times 2$ e $19 \times 8$ (note-se que também faziam a operação $19 \times 4$ como auxílio para determinar $19 \times 8$, mas este não era selecionado para a obtenção do resultado final). No final bastava somar todas as multiplicações intermédias selecionadas e obtinham o resultado. A divisão tinha um algoritmo análogo em que se ia duplicando o divisor. Este método é interessante e pode-se, por exemplo, utilizar também para observação de uma aplicação da propriedade distributiva da multiplicação em relação à adição: $19 \times 11=19 \times(1+2+8)=19 \times 1+19 \times 2+19 \times 8$.

Note-se ainda que o denominado método da multiplicação dos camponeses russos, ainda utilizado em certas regiões, é parecido ao do antigo Egipto e utiliza também duplicações e divisões sucessivas por 2 (sempre que se dividir um número ímpar neste método, deve-se desprezar a parte decimal). Contudo, tem curiosas diferenças que lhe permitem rapidamente escolher os termos a somar (basta considerar os números ímpares da coluna da esquerda e somar os números respetivos da coluna da direita). Veja-se o exemplo a seguir: 


\begin{tabular}{|r|l|}
\hline 19 & 11 \\
\hline 9,5 & 22 \\
\hline 4,5 & 44 \\
\hline 2 & 88 \\
\hline 1 & $\mathbf{1 7 6}$ \\
\hline $19 \times 11=$ & $176+22+11=209$ \\
\hline
\end{tabular}

A justificação deste método é também muito interessante e permite perceber que a divisão e a multiplicação são funções inversas uma da outra:

$$
\begin{gathered}
\mathbf{1 9} \times \mathbf{1 1}=\frac{19}{2} \times(11 \times 2)=9,5 \times 22=9 \times 22+11 \\
=\frac{9}{2} \times(22 \times 2)+11=4,5 \times 44+11=4 \times 44+22+11 \\
=\frac{4}{2} \times(44 \times 2)+22+11=2 \times 88+22+11=\mathbf{1 7 6}+\mathbf{2 2}+\mathbf{1 1}
\end{gathered}
$$

De facto, sempre que se dividem números ímpares, está-se a perder a informação relativa ao 0,5 que se despreza e, por isso, no final temos de somar esses valores ao valor final (que, no exemplo apresentado, era 176).

Um outro método muito em voga atualmente é o método das varas chinesas que permite efetuar multiplicações sem o conhecimento de qualquer tabuada (essa informação é obtida por contagem das interseções entre as varas verticais e as varas horizontais). Para compreender este método ver, por exemplo, o vídeo em

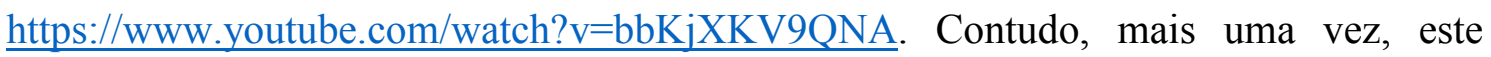
sistema é análogo ao algoritmo atual e, além disso, muito similar ao método de gelosia.

Ainda a propósito das operações aritméticas podemos referir os ábacos. Estes instrumentos são muito simples e podem facilmente ser utilizados em sala de aula. Para além do ábaco tradicional que tem 10 contas em cada coluna e que pode ser encontrado em qualquer loja de brinquedos, deve-se também introduzir o ábaco chinês onde cada coluna está dividida em duas partes: a parte de baixo tem 5 contas a valer 1 unidade cada uma, enquanto que a parte de cima tem 2 contas valendo 5 unidades cada (https://sciencing.com/use-chinese-abacus-2290925.html). Similar a este último ábaco, tem-se também o ábaco romano (https://upload.wikimedia.org/wikipedia/commons/1/13/Ábaco_banner.pdf) que é igual ao ábaco japonês (ambos têm 1 conta em cima e 4 contas em baixo). Mais uma vez, a 
comparação de diferentes instrumentos de vários povos poderá ser muito interessante em sala de aula pois permite uma visão mais universal da disciplina.

Ainda nas máquinas de cálculo, gostávamos de referir as varas de Napier (Fig. 5) que permitiam efetuar multiplicações. Estas varas foram criadas pelo matemático Napier (1550-1617) e usavam a noção já referida de multiplicação em gelosia.

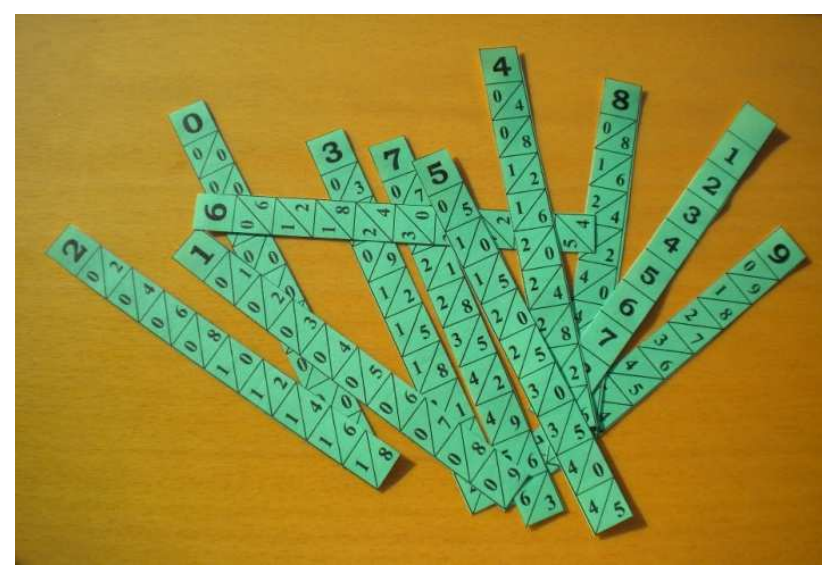

Fig. 5. Exemplo de barras de Napier

Este instrumento substituía o conhecimento das tabuadas pois cada vara continha os quadrados necessários a construir na multiplicação em gelosia. Observe-se o exemplo na Fig. 6.:

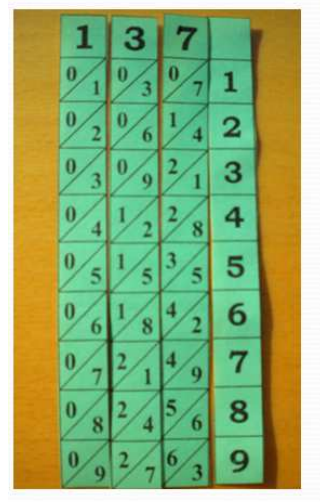

\begin{tabular}{|c|c|c|c|}
\hline $\mathbf{1}$ & 3 & 7 & \\
\hline 01 & $0 / 3$ & $0 / 7$ & 1 \\
\hline 02 & $0 / 6$ & $1 / 4$ & 2 \\
\hline 03 & $0 \%$ & $2 / 1$ & 3 \\
\hline 04 & $1 / 2$ & $2 / 8$ & 4 \\
\hline $0 / 5$ & $1 / 5$ & $3 / 5$ & 5 \\
\hline $0 / 6$ & $1 / 8$ & $4 / 2$ & 6 \\
\hline 07 & $2 / 1$ & $4 / 9$ & 7 \\
\hline 08 & $2 / 4$ & $5 / 6$ & 8 \\
\hline 0,9 & $2 / 7$ & $6 / 3$ & 9 \\
\hline
\end{tabular}

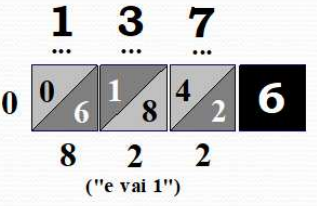

$137 \times 6=0.822$

Fig. 6. Exemplo de cálculo com as barras de Napier $(137 \times 6)$

Posteriormente, já nos finais do século XIX, criaram-se outras varas que eliminavam as principais desvantagens das varas de Napier: ser necessário fazer adições intermédias bem como, em certas situações, transportar valores para a diagonal seguinte. Essas varas designaram-se por Varas de Genaille-Lucas e são uma evolução das anteriores. Note-se que o resultado se obtém apenas e só por observação direta do instrumento, sem haver necessidade de qualquer cálculo por parte do utilizador - na coluna da direita começa-se no primeiro algarismo da linha pretendida da vara e depois 
deve-se seguir os sucessivos triângulos para as colunas adjacentes (na Fig. 7, como exemplo, encontra-se a seguinte multiplicação: 187 por 4).
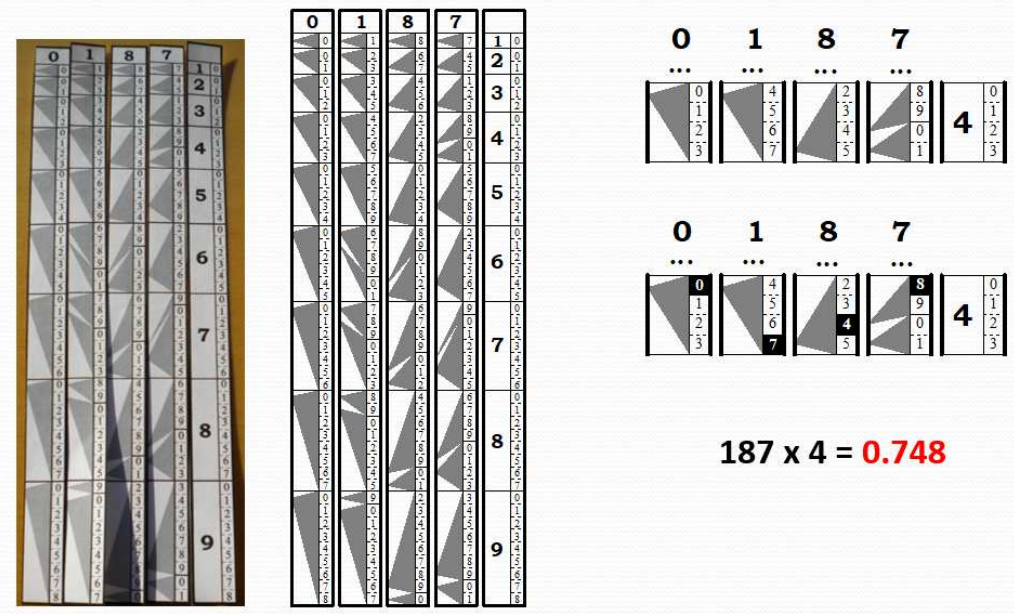

$187 \times 4=0.748$

Fig. 7. Exemplo de utilização das barras de Genaille-Lucas para o cálculo de $187 \times 4$

Não pretendemos neste ponto ser exaustivos na descrição destes instrumentos. Em (Pinto, 2011, pp. 110-117) é possível obter informações detalhadas sobre estes dois instrumentos. Serve o exposto para mostrar aos alunos que as máquinas de cálculo, tal como outros algoritmos matemáticos, foram alvo de vários séculos de evolução e de melhoramento.

\subsection{Equações}

As equações são um dos primeiros momentos da matemática escolar onde se encontra um certo grau de abstração. Note-se, contudo, que já no antigo Egito existiam problemas matemáticos que exigiam a resolução de equações. Como exemplo, considere-se o problema 26 do Papiro de Rhind:

Uma quantidade e a sua quarta parte somadas perfazem 15. Qual é a quantidade?

Em linguagem e notação atuais, diríamos que a quantidade pretendida é a solução da equação $x+\frac{x}{4}=15$ (equação do primeiro grau). O método dos antigos egípcios era substancialmente diferente do nosso, mas bastante engenhoso e designa-se por método da falsa posição (ou da falsa solução). Em primeiro lugar, os egípcios suponham que um determinado número era solução da equação (o número escolhido deveria facilitar os cálculos; no exemplo acima, o escriba escolheu o número 4 para fazer «desaparecer» a fração). Assim, com esta falsa solução, ter-se-ia: $x+\frac{x}{4}=4+\frac{4}{4}=$ 5. Mas 5 não é 15 como pretendido na equação... e, portanto, é necessário corrigir a falsa solução de que se partiu. Para o conseguir, basta notar que multiplicando 5 por 3 
se obtém o pretendido 15 e, portanto, basta fazer o mesmo à falsa solução para se obter a solução correta: $4 \times 3=12$. Este método é bastante eficaz na resolução de equações de primeiro grau (os egípcios usavam métodos similares para resolver outro tipo de equações mais complexas) e compará-lo com o atual pode constituir-se uma experiência matemática enriquecedora.

Embora as equações de segundo grau surjam bem mais tarde na matemática escolar, gostaríamos de deixar referência aos métodos de resolução utilizados historicamente pelos árabes. Estes utilizam argumentos geométricos engenhosos onde os valores das equações são representados por comprimentos de segmentos de reta e por áreas de polígonos (por exemplo, $x^{2}$ era a área do quadrado de lado $x$ ). Além disso, como ainda não existia o conceito de número negativo, os árabes tiveram de considerar diferentes métodos para diferentes tipos de equações do segundo grau.

\subsection{Geometria}

$\mathrm{Na}$ História da Geometria existem dois nomes incontornáveis e que, em nosso entender, devem ser do conhecimento de todos os professores, incluindo os dos primeiros anos: Euclides e Descartes. O primeiro, grego do século III a.C., sistematizou quase toda a geometria que se ensina atualmente nas escolas (a geometria euclidiana). $\mathrm{O}$ segundo, francês do século XVII, foi responsável pelo plano cartesiano que é essencial, por exemplo, para os sistemas de coordenadas (ensinados no $3 .^{\circ}$ ano do $1 .^{\circ} \mathrm{CEB}$ em Portugal) e para as funções.

A grande diferença entre os dois consiste na libertação da geometria cartesiana do princípio da homogeneidade. Para Euclides (assim como para os árabes quando estavam a resolver equações), a multiplicação de dois comprimentos era uma área; para Descartes, a mesma multiplicação era um comprimento de um segmento de reta. Esta diferença é marcante na História da Matemática pois permitiu um forte desenvolvimento da Geometria.

\subsection{História da Matemática local/nacional}

Em países com pouca «autoestima» como é o caso de Portugal é essencial mostrar aos alunos a produção local/nacional nesta área do saber. Em Portugal, existe muitas vezes a ideia pré-concebida por parte dos alunos que não existiu produção científica e matemática relevante no país. A este respeito podemos, por exemplo, apresentar aos alunos três momentos importantes na HM em Portugal: 
- Os aritméticos comerciais de 1500;

- Pedro Nunes e os seus tratados sobre álgebra e a navegação;

- Gomes Teixeira, um matemático internacional.

Os dois primeiros têm a vantagem de estarem relacionados com um dos momentos mais marcantes da História de Portugal - os Descobrimentos - e, além disso, permitirem o contacto com uma matemática que, à época, era verdadeiramente útil ao comércio e à navegação, duas atividades fulcrais naquele tempo. Por exemplo, os aritméticos de 1500 publicaram diversas obras matemáticas onde se abordavam vários problemas relativos à cobrança de impostos decorrentes do comércio marítimo com os «novos» mundos (para informações pormenorizadas sobre esta temática, consultar Clain, 2015). Por outro lado, a navegação portuguesa beneficiou de vários melhoramentos no problema da localização em alto-mar e que só foram possíveis com conhecimentos avançados, por exemplo, em astronomia e, em particular, em geometria esférica (observe-se, por exemplo, a curiosa questão das linhas de rumo em http://cvc.instituto-camoes.pt/ciencia/e6.html).

Gomes Teixeira, por outro lado, é um caso que permite mostrar um exemplo de uma personalidade portuguesa que se conseguiu integrar na comunidade científica internacional, surgindo o seu nome ao lado de matemáticos famosos até aos dias de hoje. Por exemplo, na Fig. 8 a seguir podemos observar o primeiro Comité de Patronage da importante revista L'Enseignement Mathématique (Furinghetti, 2003, p. 27), onde o nome de Gomes Teixeira aparece ligado a nomes tão relevantes como Poincaré, Cantor e Klein.

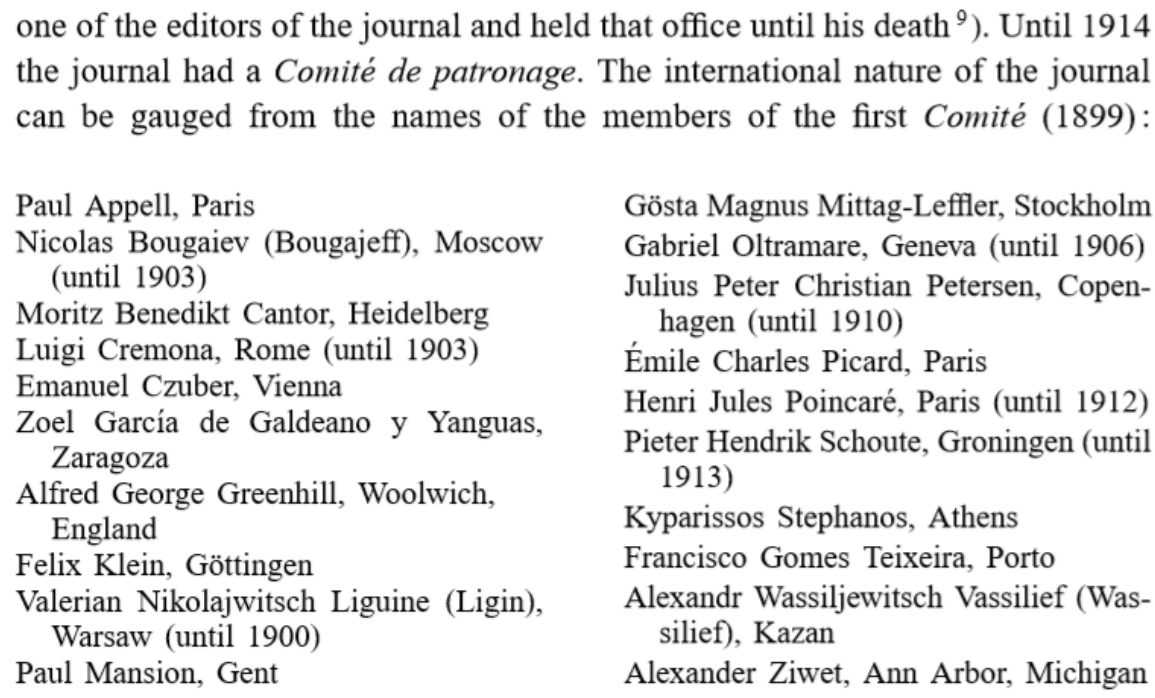

Gösta Magnus Mittag-Leffler, Stockholm Gabriel Oltramare, Geneva (until 1906) Julius Peter Christian Petersen, Copenhagen (until 1910)

Émile Charles Picard, Paris

Henri Jules Poincaré, Paris (until 1912)

Pieter Hendrik Schoute, Groningen (until 1913)

Kyparissos Stephanos, Athens

Francisco Gomes Teixeira, Porto

Alexandr Wassiljewitsch Vassilief (Wassilief), Kazan

Alexander Ziwet, Ann Arbor, Michigan

Fig. 7. O primeiro Comité de patronage da revista L'Enseignement Mathématique 
De facto, Gomes Teixeira conseguiu aproveitar o movimento de internacionalização da matemática do fim do século XIX e início do século XX para criar uma vasta rede de contactos matemáticos. Assim, o nome de Gomes Teixeira, tal como outros que se seguiram (por exemplo, Mira Fernandes, Vicente Gonçalves, e Sebastião e Silva), deve ser referenciado nas escolas, bem como na formação de professores, para que se perceba que a ciência é o produto de uma globalização, na qual Portugal também participa. O estudo da HM local é um ótimo modo de engajar os alunos com a disciplina e, para além disso, para dar a perceber que, mesmo estando num país periférico, é possível atingir-se o topo do conhecimento científico e matemático.

\section{Conclusão}

O sistema de numeração decimal atual, tal como as operações, as equações e a geometria, foi o resultado de uma longa evolução histórica. A sua escolha deve-se a diversas qualidades que só podem ser cabalmente compreendidas quando este sistema é comparado com outros sistemas antigos atualmente em desuso. Para diversos povos antigos a matemática foi essencial para o desenvolvimento de áreas relacionadas como a astronomia, a navegação, a engenharia, a cobrança de impostos, etc., tendo o mesmo ocorrido na História de Portugal onde se destacam os conhecimentos marítimos e os aritméticos ligados ao comércio. O conhecimento destes factos permite uma humanização da matemática que não seria possível de outro modo. Assim, somos da opinião que a HM deve ser uma das ferramentas educativas que todo o professor da Educação Básica deve possuir no seu arsenal de conhecimentos. Note-se, contudo, que esta ferramenta não deve ser exclusiva, mas sim complementar a outras ferramentas como, por exemplo, o uso da tecnologia e da matemática recreativa em sala de aula. Cada professor deve possuir várias e diferenciadas estratégias de que se possa socorrer e utilizar em diferentes contextos e situações. Alargando e aprofundando deste modo o seu conhecimento de conteúdo sobre tópicos de HM, em particular os que apresentamos, bem como adquirindo ferramentas uteis ao desenvolvimento do seu conhecimento pedagógico de conteúdo.

Com a utilização da HM em sala de aula mostra-se que a matemática é um corpo de saber proveniente de várias latitudes começando por quebrar a ideia pré-concebida que a matemática (tal como todo o restante conhecimento científico) é um produto exclusivo da atual civilização ocidental. Numa época em que os radicalismos 
aumentam, este conhecimento de HM pode contribuir para um maior respeito e uma maior tolerância para com a cultura e o saber de outros povos diferentes dos ocidentais. Note-se, por fim, que a utilização da HM como ferramenta pedagógica é uma área científica bem estabelecida, tendo o seu expoente máximo no grupo internacional «HPM- International Study Group On the History And Pedagogy of Mathematics». O grupo HPM, criado em 1976, é um afiliado do ICMI, a mais importante organização internacional dedicada à educação matemática. Para uma primeira abordagem aos trabalhos científicos deste grupo, bem como a uma lista de eventos passados e futuros consultar o website http://www.clab.edc.uoc.gr/hpm/about\%20HPM.htm.

\section{Bibliografia}

Arcavi, A., Bruckheimer, B., \& Ben-Zvi, R. (1982). Maybe a mathematics teacher can profit from the study of the history of mathematics. For the Learning of Mathematics, 3(1), 30-37.

Boyer, C. (1996). História da Matemática. São Paulo: Editora Edgard Blucher.

Clain, T. C. (2015). A matemática e o comércio em Portugal através das obras de aritméticos do século XVI: Gaspar Nicolas, Ruy Mendes e Bento Fernandes (Tese de Doutoramento). Aveiro: Universidade de Aveiro.

Estrada, M. F., Sá, C. C., Queiró, J. F., Silva, M. C., \& Costa, M. J. (2000). História da Matemática. Lisboa: Universidade Aberta.

Eves, H. (1997). Introdução à História da Matemática (2. ${ }^{\circ}$ ed.). Campinas: Editora da UNICAMP.

Furinghetti, F. (2003). Mathematical instruction in an international perspective: the contribution of the journal L'Enseignement Mathématique. In Coray, D., Furinghetti, F., Gispert, H., Hodgson, B. R. \& Schubring, G. (Ed.), One Hundred Years of L'Enseignement Mathématique. Geneve: L'Enseignement Mathématique.

Galante, D. (2014). The Use of the History of Mathematics in the Teaching Pre-service Mathematics Teachers. REDIMAT, 3(2), 110-120. doi: 10.4471/redimat.2014.45

Galvão, C., \& Ponte, J.P. (2018). Os Mestrados em Ensino no Contexto Atual da Formação de Professores em Portugal. In C. Galvão \& J. P. Ponte (Org.), Práticas de Formação Inicial de Professores: Participantes e Dinâmicas (pp. 13-24). Lisboa: Instituto de Educação da Universidade de Lisboa. 
Galvão, C., Ponte, J.P., \& Jonis, M. (2018). Os Professores e a sua Formação Inicial. In C. Galvão \& J. P. Ponte (Org.), Práticas de Formação Inicial de Professores: Participantes e Dinâmicas (pp. 25-46). Lisboa: Instituto de Educação da Universidade de Lisboa.

Isaacs, I., Ram, V. M., \& Richards, A. (2000). A historical approach to developing the cultural significance of mathematics among first year preservice primary school teachers. In V. Katz (Ed.), Using history to teach mathematics - An international perspective. MAA Notes (No. 51, pp. 123-128). Washington: The Mathematical Association of America.

Jankvist, U.T., Clark, K.M., \& Mosvold, R. (2019). Developing mathematical knowledge for teaching teachers: potentials of history of mathematics in teacher educator training. J. Math Teacher Educ. https://doi.org/10.1007/s10857-018$\underline{09424-\mathrm{x}}$

Katz, V. (2004). The History of Mathematics. Brief Edition. Boston: Person Education Inc.

Lima, E., Pedroso, N., Barrigão, N., \& Santos, S. (2019). Alfa Matemática 3. Porto: Porto Editora.

Mendes, I. A. (2015). História da Matemática no Ensino. São Paulo: Editora Livraria da Física.

Mendes, I. \& Chaquiam, M. (2016). História nas aulas de Matemática: fundamentos e sugestões didáticas para professores. Belém: SBHMat.

Mosvold, R., Jakobsen, A., \& Jankvist, U.T. (2014). How Mathematical Knowledge for Teaching May Profit from the Study of History of Mathematics. Sci \& Educ, 23, 47-60. DOI 10.1007/s11191-013-9612-7

National Council for the Accreditation of Teacher Education. (2003). NCATE/NCTM Program standards for programs for initial preparation of mathematics teachers. Washington, DC: Author.

Pinto, H. (2011). História da Matemática na sala de Aula. Lisboa: Ludus.

Shulman, L. S. (1986). Those Who Understand: Knowledge Growth in Teaching. Educational Researcher, 15(2), 4-14.

Struik, D. (1992). História Concisa das Matemáticas. Lisboa: Gradiva. 


\section{Agradecimentos}

Este trabalho é financiado por Fundos Nacionais através da FCT - Fundação para a

Ciência e a Tecnologia, I. P., no âmbito do projeto UID/CED/00194/2019 e do projeto UID/MAT/04106/2019 e ainda pelo CIDMA - Centro de Investigação e Desenvolvimento em Matemática e Aplicações.

Autores

Hélder Pinto

Instituto Piaget, RECI - Research in Education and Community Intervention e CIDMA -

Centro de Investigação e Desenvolvimento em Matemática e Aplicações. ORCID: https://orcid.org/0000-0002-2226-0685. E-mail: helder.pinto@gaia.ipiaget.pt

\section{Cecília Costa}

Universidade de Trás-os-Montes e Alto Douro e CIDTFF - Centro de Investigação em Didática e Tecnologia na Formação de Formadores (Lab-DC\&T na UTAD). ORCID: https://orcid.org/0000-0002-9962-562X. E-mail: mcosta@utad.pt 